\title{
TUTORÍA ACADÉMICA EN LA EDUCACIÓN SUPERIOR: EL ROL DEL AUTOR ACADÉMICO, TUTOR PEDAGÓGICO Y DEL ESTUDIANTE EN LA MODALIDAD A DISTANCIA
}

\author{
ACADEMIC TUTORING IN HIGHER EDUCATION, \\ DISTANCE MODALITY: THE ROLE OF THE ACADEMIC \\ AUTHOR, PEDAGOGICAL TUTOR AND THE STUDENT IN \\ THE DISTANCE MODALITY
}

\author{
Aracelly Fernanda Núñez Naranjo ${ }^{1}$ \\ Recibido: 2021-06-20 / Revisado: 2021-07-16 / Aceptado: 2021-08-12 / Publicado: 2021-09-15
}

Forma sugerida de citar: Núñez-Naranjo, A. F. (2021). Tutoría académica en la educación superior: el rol del autor académico, tutor pedagógico y del estudiante en la modalidad a distancia. Retos de la Ciencia. 5(e). 64-75. https://doi.org/10.53877/rc.5.e.20210915.06

\section{RESUMEN}

La tutoría académica constituye una práctica pedagógica que permite la construcción del conocimiento para los diferentes actores involucrados en el proceso, es así, que este trabajo reflexiona sobre la tutoría académica en la educación superior, en las carreras de educación con una modalidad a distancia, conformados por tres sujetos, el autor académico, el tutor pedagógico y el estudiante. Este análisis, parte de un conjunto de cuestionamientos sobre cada uno de los diferentes roles, así como, menciona las realidades situadas, la finalidad en el entorno y medio social virtual e identifica cuatro dimensiones, la pedagógica, la administrativa, la tecnológica y la social, las que son intervinientes en el proceso de enseña y aprendizaje, que tiene como propósito el aprender a comprender, aprender a ser, aprender a convivir, aprender a actuar y aprender a decidir acercándose a la realidad como actor en su rol y posibilitando el trabajo autónomo y colaborativo. Concluye la tutoría académica requiere de un proceso de reflexión en torno a la meta de formación personal, profundización del rol, la identificación de las necesidades de los estudiantes, las metas académicas, aspectos emocionales en las que las dimensiones facilitan el proceso de enseñanza y aprendizaje.

Palabras clave: autor académico, educación superior, enseñanza y aprendizaje, modalidad a distancia, tutoría académica, tutoría, tutor pedagógico.

\footnotetext{
${ }^{1}$ Magister en Gestión de Proyectos Socio Productivos. Docente en la Facultad de Ciencias Humanas de la Educación y Desarrollo Social, Carrera de Educación Básica; Docente en la Maestría en Educación Mención Innovación y Liderazgo Educativo, Especialista en Pedagogía Universidad Tecnológica Indoamérica. Ecuador. Email: fernandanunez@indoamerica.edu.ec / ORCID: https://orcid.org/0000-0001-7431-2339
} 


\section{ABSTRACT}

Academic tutoring constitutes a pedagogical practice that allows the construction of knowledge for the different actors involved in the process, thus, this work reflects on academic tutoring in higher education, in education careers with a distance modality, made up of three subjects, the academic author, the pedagogical tutor and the student. This analysis, based on a set of questions about each of the different roles, as well as mentions the realities located, the purpose in the environment and virtual social environment and identifies four dimensions, the pedagogical, the administrative, the technological and the social, which are involved in the process of teaching and learning, which has as its purpose to learn to understand, learn to be, learn to live together, learn to act and learn to decide approaching reality as an actor in your role and enabling autonomous and collaborative work. Academic tutoring requires a process of reflection on the goal of personal formation, deepening of the role, identification of the needs of students, academic goals, emotional aspects in which the dimensions facilitate the teaching and learning process.

Keywords: academic author, higher education, distance modality, pedagogical tutor, tutoring, academic tutoring, teaching and learning.

\section{INTRODUCCIÓN}

Las instituciones de educación superior públicas, privadas o cofinanciadas han buscado alternativas para reforzar los conocimientos en sus estudiantes incluyendo programas tutoriales que permitan elevar el nivel educativo pretendiendo la mejora no solo en el rendimiento académico sino en la construcción del conocimiento (Maluenda, Moraga y Díaz, 2019; Gómez-Collado, 2012), siendo uno de los grandes retos de la educación superior formar a los profesionales para que sean creativos, eficientes y eficaces en el desempeño de sus funciones con responsabilidad social y con el medio ambiental que los rodea (Salazar-Gómez, Tobón y Juárez-Hernández, 2018).

Los países iberoamericanos, han sido influenciados por el espacio europeo de educación superior en el siglo XX. Para el año 2002 en Latinoamérica, se realizaron transformaciones en la educación, alcance de metas y objetivos mediante proyectos para trabajar de manera similar con las instituciones europeas promoviendo la sociedad del conocimiento y el desarrollo sostenible, cinco años después, se alinean con proyectos que tienen como base el intercambio académico, científico, equipamiento, entre otros (Lobato Fraile y Guerra Bilbao, 2016; Franco, 2017), sin embargo en algunas modalidades de estudio, se presentan diferentes factores que requieren intervención como: incremento de estudiantes carreras nuevas, altas tasas de deserción, rezagamiento académico, repitencia y baja titulación, (Núñez-Naranjo, 2020; Badillo-Guzmán, 2007), de ello parten los cambios estructurales organizativos de las instituciones de educación superior, en los que se evidencian a los actores docentes y estudiantes y en los que inician intervenciones mediante estrategias para fortalecer la permanencia, la titulación, la construcción del conocimiento y rendimiento académico, mediante la enseñanza y el aprendizaje (Núñez-Naranjo, 2019), con un acompañamiento centrado en las necesidades del estudiante mediante la tutoría.

La acción tutorial, por su misma función de tutelar, ha sido utilizada por docentes a lo largo de la historia con el apoyo de diferentes medios tecnológicos y herramientas entre los más conocidos son la tutoría presencial, por correspondencia, telefónica, 
telemática y tecnológica. (Pagano, 2008) La figura del tutor en la modalidad a distancia aparece para responder a las necesidades educativas en el ámbito virtual o en línea, ya que el tutor, se convierte en un orientador (Sánchez, 2015). Sin embargo, la figura del docente en la función educativa superior en la modalidad a distancia presenta confusión entre los roles del autor académico y tutor pedagógico.

Autores como Gonzalo (2012) y García-Valcárcel (2008), mencionan que en la modalidad a distancia las universidades declaran la existencia de dos tipos de tutorías académicas: individuales y grupales, estas tutorías permiten reforzar algún contenido, mejorar el rendimiento académico o brindar retroalimentación por inasistencia a clases, a ello se suma García y otros (2015) indicando que esta tutoría académica forma parte del proceso de acompañamiento y guía al estudiante durante todo su proceso de formación incluida la elaboración de su trabajo final para su graduación.

La tutoría académica en la modalidad a distancia, se ha convertido en un escenario de preocupación tanto para tutores como para directivos, esta situación está asociada a dificultades que conllevan escasa interacción entre el personal académico y el estudiante, espera de largos tiempos en recibir la retroalimentación de tareas, evaluación, etc, poco acceso y uso de la tecnología, incomprensión de tareas, dificultades en la obtención del material de estudios, entre otros (Hernández, Tobón, Gonzalez y Guzmán, 2015). Este proceso de gestión de tutorías está relacionado a cuatro dimensiones pedagógicas, administrativas, sociales y tecnológicas (Ponce Ruiz, 2021)

En las carreras a distancia la tutoría académica presenta poca aceptación o interés por parte del estudiante, se ha identificado a alumnos que al ingresar a la tutoría académica dedican su tiempo a realizar actividades distintas, la misma modalidad en su política gubernamental, no establece la asistencia a la tutoría académica como obligatoria, lo que acarrea un ingreso bajo a la clase, así como la escasa atención a la explicación realizada por el docente en tiempo real, con este antecedente, se realizan las siguientes preguntas de reflexión: ¿para qué ingresar a una tutoría si no va a atenderla?, ¿qué trata de dar a entender el estudiante al docente?, ¿las tutorías realizadas no son de interés para el dicente?, ¿por qué el estudiante cree importante, se vea su nombre en las clase en el aula virtual?, ¿cuánto afecta al aprendizaje y lo evidencia en su titulación?

Estas interrogantes se confrontan desde los ámbitos docente, dicente, recursos tecnológicos y desde el mismo curriculum. Si bien, el docente en la tutoría brinda al estudiante pautas importantes para el desarrollo de sus actividades autónomas y lo guía para el fortalecimiento de sus competencias además de suplir interrogantes en la actividad presencial virtual, también es cierto que el estudiante puede autogestionar su aprendizaje, a ello, Paulo Freire menciona "quien forma se forma y reforma al formar y quien es formado se forma y forma al ser formado" (Freire, 1996) he ahí la importancia de una tutoría académica, la misma práctica permite un aprendizaje mutuo, duradero y gratificante para los actores docente y estudiante, pero para ello es indispensable la interacción, la comunicación asertiva y aún más importante el reconocimiento de saberes previos, estos involucran un proceso integral desde el inicio hasta la finalización de su formación profesional (Obaya y Vargas, 2014).

A fines del 2019, la pandemia ocasionada por COVID-19, influyó en la educación, más que en los procesos de enseñanza, tanto fue su impacto que el sistema educativo dio un giro en el que las instituciones de educación superior tuvieron que cambiar o modificar sus procesos de educativos. A ello este involucramiento y mayor uso de los recursos tecnológicos trajo consigo la necesidad urgente del desarrollo de destrezas 
y habilidades tecnológicas, siendo la acción tutorial un medio que delimitó la calidad de la formación integral en el medio virtual y posibilitó la orientación para que los estudiantes y docentes se relacionen entre sí y con los medios virtuales y tecnológicos (Chacón, 2021; Diaz, Iglesias y Valdez, 2020).

\section{DESARROLLO}

Desde una mirada al rol del docente.- en la educación a distancia este rol conlleva una guía y acompañamiento al estudiante sobre el aprendizaje, es un actor activo que crea "posibilidades de producción o construcción de conocimientos" (Freire, 1996), de esta manera ¿qué comprende la posibilidad de producción?, ¿qué comprende este acompañamiento y guía para la construcción del conocimiento?, desde estas preguntas el panorama académico conlleva no solo un conjunto de saberes a ser transmitidos, sino un proceso motivacional, metacognitivo en el que se aplican recursos didácticos y metodológicos que permiten la construcción de nuevos aprendizajes partiendo de uno anterior o el fortalecimiento de estos.

Enseñar entonces, tiene un significado más profundo que solo transferir conocimientos, enseñar una asignatura, enseñar contenidos, más que el solo hecho de formar. Enseñar es crear esas posibilidades de construcción y reconstrucción, en el que el estudiante puede construir su propia incertidumbre que le permita y motive un nuevo aprendizaje y que este sea significativo, por tanto no hay docencia sin dicencia, no hay enseñar sin aprender, ni aprender sin enseñar, así quien enseña aprende y quien aprende enseña al aprender (Freire, 1996), por ello no es lo mismo memorizar un conjunto de saberes si no va a existir una reflexión crítica sobre ello, el intelectual memorista repite, el intelectual reflexivo crea y permite ambientes de construcción.

Desde la perspectiva anterior en el que el docente tiene funciones específicas en las que busca guiar a los estudiantes, el estudiante tiene también un rol. En la educación a distancia sobre todo en la pandemia, el rol del dicente juega un papel fundamental a la hora de aprender y enseñar, pues los contextos son diferentes. Si se enseña cómo se aprendió hace algunas décadas en las que la memoria era lo primordial y solo existía un padre del conocimiento el docente, entonces, continuaríamos en el mundo de la enseñanza bancaria, por ello romper estos paradigmas cartesianos conductistas conlleva a involucrar nuevos saberes, a realizar una reflexión crítica sobre la práctica, y quizá de esta manera el dicente logrará estimular su curiosidad nata de la que forman parte la comprobación, validación, comparación sobre un nuevo conocimiento.

En la modalidad a distancia, se conoce dos tipos de actores intervinientes en el proceso de enseñanza, que no siempre tiene resultados positivos, el autor académico y el tutor pedagógico, a ello, ¿quién es el tutor pedagógico?, ¿quién es el autor académico?, ¿qué tipo de relación mantienen en el proceso de enseñanza?, no es fácil llegar a ser un docente problematizador, pues es más fácil seguir el curriculum formal sin antes conocer al estudiante, mientras que en la práctica es el curriculum real ese enseñado y ese aprendido el que hace la diferencia o el que permite el desarrollo de un nuevo líder, de un emprendedor. Es menos enriquecedor que el docente cambie de tema cuando en la tutoría se desconoce sobre un punto específico, así este curriculum oculto, oculta también las falencias del educador bancario, he ahí ese rompimiento entre el autor académico, el tutor pedagógico y el estudiante. 
Llegar a obtener un título en la actualidad se ha vuelto un desafío, asociado a variables que en el caminar influyen de manera positiva o negativa en la decisión de los estudiantes por permanecer o desertar (Núñez-Naranjo, 2019; Quisel y Campana Concha, 2020), lo que conlleva no solo un conjunto de factores personales, sociales, políticos, sino involucra el proceso mismo del acompañamiento, guía y motivación desde el área académica (Núñez-Naranjo, 2020).

En este sentido, ¿qué perfil del educador se busca?, ¿qué habla nuestro modelo educativo, pedagógico y curricular sobre este perfil?, se han establecido dentro de la normativa universitaria un conjunto de responsabilidades y competencias que el maestro requiere para enseñar, es así que se establecen actitudes y aptitudes en torno a implementar prácticas pedagógicas orientadas a la creación de la sociedad del conocimiento al desarrollo social sostenible, a la resolución de problemas reales del contexto por medio de la aplicación metodológica de proyectos, por medio de una evaluación continua, retroalimentación por criterios, instrumentos y mecanismos de evaluación (Tapia, Lara, Aranda y Naranjo, 2018; Universidad Tecnológica Indoamérica, 2018), más allá de ello, ese educador debe ser innovador, indagador e instigador creando ambientes de enseñanza que posibiliten el aprendizaje crítico.

Rol del tutor pedagógico: El docente tutor por normativa gubernamental presenta un rol en la modalidad a distancia en que se menciona como apoyo orientador, acompañante, motivador sobre el autoaprendizaje (CES, 2020), está entonces estrechamente asociado al proceso mismo de la motivación, retroalimentación adecuada y guía permanente (Gairín, Feixas, Guillamón y Quinquer, 2004), en la que interviene una comunicación asertiva tanto con el estudiante como con el autor. Es así como la tutoría debe tomar un papel motivante (Ver Figura No. 1). En este rol se involucra no solo una calificación o retroalimentación por medio de la rúbrica, sino además debe conllevar claramente a: responder preguntas de manera asertiva, aclarar dificultades, crear condiciones de aprendizaje colaborativo de forma efectiva y eficiente (Floris y Guidi, 2010; Mele, 2017; García-Valcárcel, 2008), es quien debe estar cercano al estudiante, orientarlo, acompañarlo y guiarlo.

El tutor pedagógico, debe tener habilidades de generen un ambiente armónico colaborativo y motivante mediante la moderación en diferentes actividades de acompañamiento que permitan enriquecer el conocimiento de los estudiantes, pues requiere además de un conjunto de atributos que le permitirán un mejor acercamiento. (Conesa Sánchez, 2017; Medina Alfonso, 2015), asimismo, como señalan Castillo y Montoya (2015) las acciones formativas en el contexto escolar comunitario son conscientes e intencionadas, el docente aprovecha sus propias experiencias para mejorar progresivamente la intervencion en la formacion de seres humanos. (p. 7)

Rol del autor académico: El docente autor académico en normativa gubernamental establece que el responsable de la asignatura es quien debe implantar estrategias de aprendizaje, seguimiento y evaluación (CES, 2020). En esta modalidad debe presentar una comunicación in-situ por medio de la tutorías en ambientes de aprendizaje virtuales, estableciendo una comunicación que facilite el crecimiento del conocimiento científico y académico, preparando al estudiante para el dominio del curriculum que le permita alcanzar su perfil de egreso (Ver Figura 1), para ello indispensable la aplicación de estrategias didácticas que fomenten la curiosidad y su motivación en su acción formativa de orientar, ayudar y mediar (Saiz, 2021; Pagano, 2008). El autor académico pasa a ser, de un sujeto transmisor de contenidos a uno orientador por medio de la interacción individual y social como mediador de los ambientes para orientar al estudiante (Franco, 2017; Silva, 2010). 


\section{Figura 1:}

Rol del autor académico y tutor pedagógico

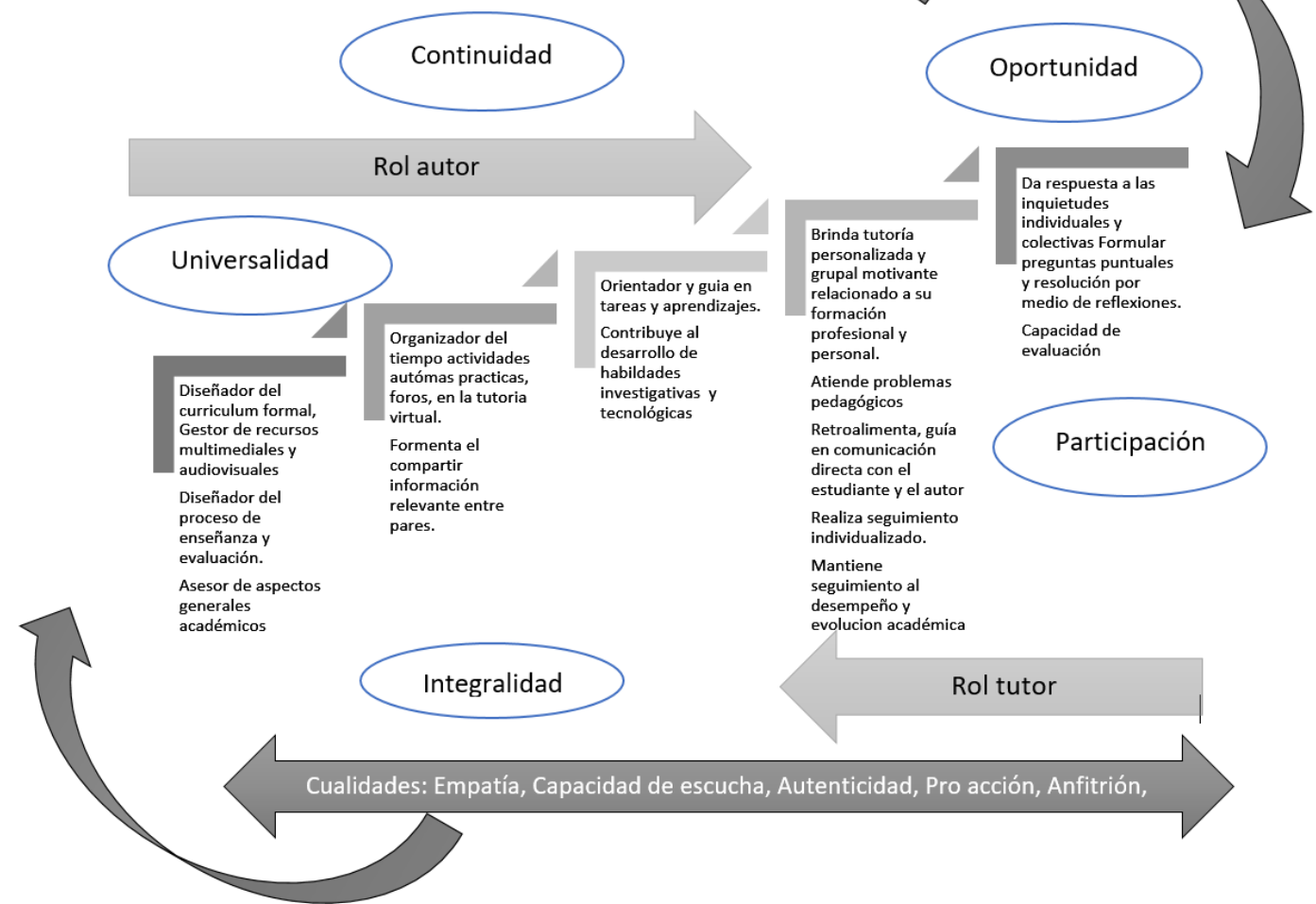

Nota: La figura representa el rol del autor académico y rol del tutor pedagógico y sus cualidades

Autor académico y Tutor pedagógico: Tanto el autor académico como el tutor pedagógico deben coordinar acciones de enseñanza y aprendizaje que permitan una integralidad de los procesos, la participación de los estudiantes y los dos actores, por medio de la universalidad, continuidad y oportunidad, enfocados en metas académicas, en aspectos emocionales y de adaptación que forman parte de su proceso de formación, ser facilitadores del aprendizaje, diseñadores de situaciones mediadas del aprendizaje, orientadores y evaluadores continuos (Ver Figura 1).

Rol del estudiante: se busca que el estudiante se motive por medio de su propia autogestión de aprendizaje, que desarrolle su capacidad crítica, propositiva, reflexiva, que por medio del saber ser, hacer, conocer sea capaz de construir y reconstrucción de conocimiento (García-Valcárcel, 2008). El estudiante en su rol debe tener liderazgo, ese liderazgo que le permita resolver problemas reales de entorno, tomar decisiones. 
Figura 2:

Rol del estudiante

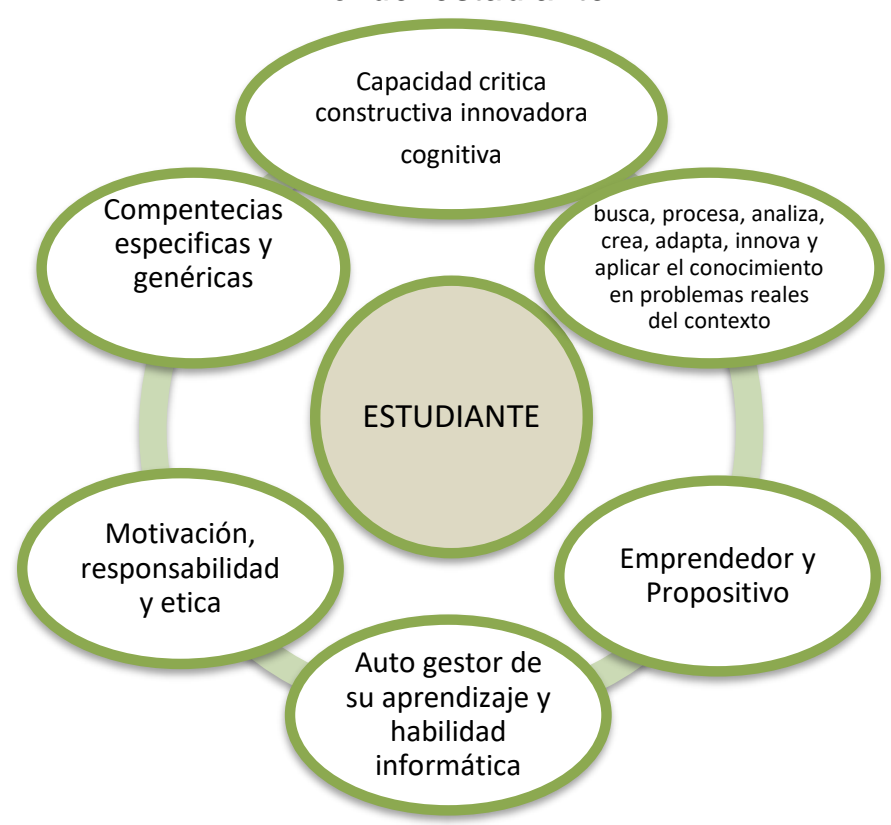

Nota: La Figura, representa el rol del estudiante en la modalidad a distancia Fuente: Elaboración propia

Es indispensable que los estudiantes comprendan y asuman su rol, (Ver Figura 2) y con ello, sean capaces de identificar los problemas del contexto que los rodea, sean capaces de interpretarlos argumentarlos y resolverlos en el que se involucren un trabajo colaborativo y teniendo experiencias vitales para formar y consolidar los valores universales (Tobón, 2014).

Se busca entonces que el estudiante aprenda. Este aprender consiste en 5 dimensiones a las que conlleva la tutoría académica que va más allá de la impartición de una clase, que la impartición de un contenido y del desarrollo de competencias específicas o genéricas de un perfil de egreso, según Marrero Sánchez, Mohamed Amar y Xifra Triadú (2018), Cabalín Silva y Navarro Hernández (2008), Salas (2000) entre ellas se requiere:

\section{Tabla 1:}

Dimensiones de la Tutoría Académica

Aprender a Consiste en comprender el mundo que lo rodea.

comprender Identificar la problemática en un contexto determinado o en contextos similares

Problematizar el conocimiento y abordar problemáticas sociales en armonía con el medio ambiente.

Aprender a Consiste en construir su propia identidad, conocerse a sí mismo, su ser ideología, su imagen, dentro de una vertiente personal y otra social

Aprender a Consiste en que se armoniza con el medio que lo rodea, por medio del convivir respeto a su prójimo así mismo y a la naturaleza.

Aprender a Consiste en que identifique sus principales fortalezas y debilidades actuar permitiéndose crear oportunidades de reflexión, diálogo en relaciones sociales, profesionales y laborales

Aprender a Consiste en que pueda tomar sus propias decisiones fundamentadas en la decidir búsqueda del bien común con criterio.

Nota: Dimensiones de la tutoría académica 
En la tutoría dentro del rol del autor académico es necesario una aportación significativa, en la que intervienen cuatro dimensiones: aspectos tecnólogos, administrativos, sociales y pedagógicos, desempeñando distintas funciones que suman al proceso de enseñanza y aprendizaje, estas dimensiones posibilitan el trabajo autónomo y colaborativo (Ver Figura 3).

Figura 3:

Dimensiones

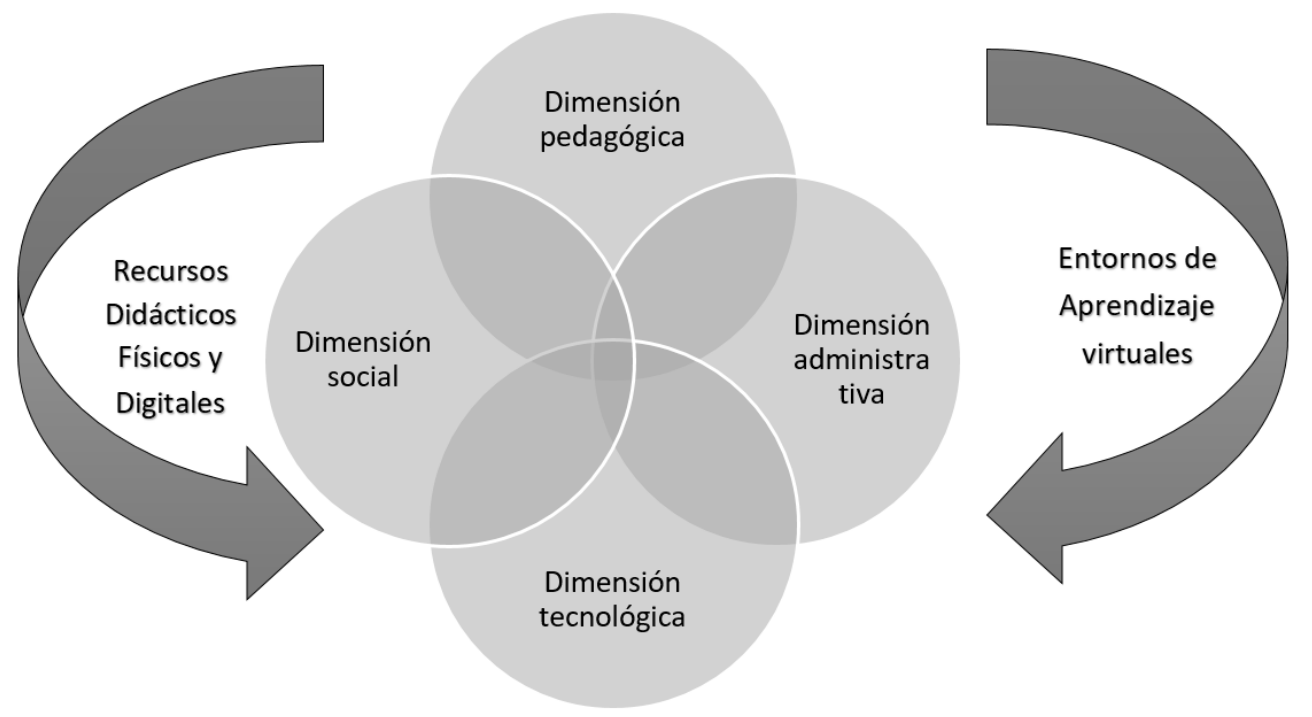

Nota: La figura representa las 4 dimensiones de la tutoría académica en la que los entornos virtuales y recursos didácticos, físicos y digitales son intervinientes.

Dimensión Pedagógica. - Esta dimensión refiere a la capacidad en el punto educativo necesarios para el proceso de enseñanza y aprendizaje en la modalidad a distancia, pues hace referencia a propósitos, a los contenidos, a la metodología, a la didáctica, en sí a hace referencia a las prácticas pedagógicas cotidianas (Espinoza Freire y Ricaldi Echevarría, 2018; Conesa Sánchez, 2017; Garduño Vera, 2007). En esta dimensión se enfatiza la función que debe realizar la institución de educación superior, en un ambiente de en el que se construyan conocimientos, identificando las necesidades de los estudiantes.

Dimensión Tecnológica. - Esta dimensión corresponde al conocimiento, habilidades y destrezas que se poseen entorno al uso de la tecnología, en el que se debe ser capaz de crear ambientes virtuales de aprendizaje con un clima agradable (Espinoza Freire y Ricaldi Echevarría, 2018) para que el estudiante sienta gusto con su proceso de aprendizaje y el entorno virtual que lo rodea.

Dimensión Administrativa. - Articulación entre la dimensión pedagógica y tecnológica gestora del proceso de enseñanza y aprendizaje (Espinoza Freire y Ricaldi Echevarría, 2018). Esta dimensión constituye la articulación de las personas con los equipos de trabajo y los recursos, con ello permiten administrar el tiempo, aprovechar recursos disponibles y construir ambientes de comunicación asertiva. Este aprovechamiento de las dimensiones tecnológica y pedagógica conlleva a generar nuevas propuestas de enseñanza, estrategias, en aras de una mayor permanencia y titulación.

Dimensión Social. - Esta dimensión trata sobre la creación de entornos virtuales amigables, manejo y uso de herramientas multimediales y audiovisuales que permitan 
la interacción individual y colectiva (Espinoza Freire y Ricaldi Echevarría, 2018; Conesa Sánchez, 2017). La vinculación del estudiante con el docente y los medios virtuales permite una cooperación, participación y aprovechamiento de recursos que ofrecen las IES.

Con la finalidad de que la tutoría académica sea un entorno de compartir y crear conocimientos, es necesario que se aborde temáticas como parte de la inducción a la vida universitaria, lo que permitirá comprender qué y cómo estudiar en la modalidad a distancia, actitudes, hábitos y uso de la tecnología (Floris y Guidi, 2010), temas como:

Aprendizaje en entornos virtuales: EVA's, diseñadas con finalidades formativas, con un espacio estructurado pero dinámico. EVA's, con espacios sociales de crecimiento profesional y personal, en el que el estudiante sienta confianza, sensación de pertenencia a la comunidad universitaria, sensación de realización profesional (Silva, 2010).

Inducción a la vida universitaria a distancia: Para ello una inserción a la vida universitaria debe ser planificada y ejecutada con responsabilidad académica, en el que se involucra, no un conjunto de contenidos y uso de EVA's, sino el hacer que el estudiante se sienta parte de la institución, en el que se le proporcione pautas motivadoras.

Reconocimiento de deberes, responsabilidades y obligaciones estudiantiles: Una de las principales acciones a ser considera por las instituciones que ofertan modalidades a distancia requiere de la sensibilización en el estudiante entorno al reconocimiento como sujeto de aprendizaje y enseñanza, en el que reflexione frente a su rol estudiantil.

Los recursos tecnológicos y su aplicación profesional: Para ello el docente debe tener conocimientos solidos sobre el uso de los diferentes recursos multimediales y audiovisuales, ofreciendo esa posibilidad de un ambiente social como objeto del aprendizaje virtual. El docente debe ser el creador de su enseñanza, con el uso de tecnológica un ambiente virtual favorable.

Autogestión del aprendizaje: Fomentar la autogestión del aprendizaje en la modalidad a distancia, por medio de la independencia, responsabilidad, motivación por aprender y prepararse, para ello se requiere de planificación, seguimiento, control y evaluación (Núñez Naranjo, Becerra García y Olalla Pardo, 2021). Los estudiantes que ingresan a la modalidad a distancia deben fortalecer su independencia y responsabilidad académica, además de plantearse metas y propósitos claros que los motive a persistir y culminar su carrera.

\section{CONCLUSIONES}

El sentido de la tutoría académica requiere de un proceso de reflexión en torno a la meta de formación en la que intervienen tres actores que facilitan el proceso de enseñanza y aprendizaje, para que se logren los objetivos de formación académica esperada es necesario que exista claridad en las funciones establecidas de acuerdo a cada rol pues involucra una coordinación entre el autor académico y tutor pedagógico así como responsabilidades del estudiante, pues se requiere de un involucramiento con motivación y ética que le permita construir y reconstruir los conocimientos, aportando a su formación académica, a su aprendizaje y a su rol en su vida profesional. 
Es necesario una profundización del rol del autor académico y del tutor pedagógico y de las acciones individuales que suman al acompañamiento y guía del estudiante, de la misma manera la es necesaria la identificación de las necesidades de los estudiantes, en las que interviene las dimensiones pedagógica, administrativa, tecnológica y social así como también la universalidad, continuidad y oportunidad, que involucran las metas académicas, los aspectos emocionales y la adaptación como proceso de formación.

La universidad, requiere de un proceso inducción fortalecido desde la misma inducción a la educación superior en la modalidad a distancia, ya que es la primera impresión que el estudiante tendrá en cuanto a sus expectativas académicas, dicho de esta menara el abordaje al ingreso a la vida universitaria requiere de un énfasis en el aprendizaje en los entornos virtuales en la modalidad a distancia, el reconocimiento de deberes, responsabilidades y obligaciones estudiantiles, recursos tecnológicos y su aplicación profesional siendo necesario además, lo establecido por Núñez Naranjo, Becerra García y Olalla Pardo (2021) la autogestión de su propio aprendizaje.

\section{REFERENCIAS BIBLIOGRÁFICAS}

Badillo Guzmán, J. (2007). La tutoría como estrategia viable de mejoramiento de la calidad de la educación superior. Reflexiones en torno al curso. Revista de Investigación Educativa, $1-22$ Obtenido de https://www.redalyc.org/pdf/2831/283121712006.pdf

Castillo Bustos, M. y Montoya Rivera, J. (2015). Dinamica ideo-espiritual de la formacion estetico-pedagogica del docente. Alteridad, 10(2), pp. 190-204.

CES. (2020). Reglamento de Régimen Académico. Quito. Recuperado el 15 de 05 de 2021, de gaceta.ces.gob.ec

Conesa Sánchez, S. (25 de 05 de 2017). El papel del tutor a Distancia. Obtenido de Campus educación.: https://www.campuseducacion.com/blog/revista-digitaldocente/el-papel-del-tutor-a-distancia/

Chacón, M. (2021). Acción tutorial en el fortalecimiento del perfil profesional universitario: aportes en el desarrollo de competencias a partir de la educación virtual. Espacios, 42(5), 66-77. doi:DOI: 10.48082/espacios-a21v42n05p05

Diaz, J., Iglesias, M., \& Valdez, M. (2020). La tutoría a distancia: acciones del tutor en la Estrategia de Formación Doctoral en tiempos de COVID 19. Scielo, 18(3), 478-484. doi:versión On-line ISSN 1727-897X

Espinoza Freire, E., \& Ricaldi Echevarría, M. (2018). El tutor en los entornos virtuales de aprendizaje. Scielo, 10(3), 201-210. Obtenido de http://scielo.sld.cu/scielo.php?script=sci_arttext\&pid=S221836202018000300201

Floris, C., \& Guidi, M. (2010). Curso de ingreso virtual para educacion virtual: una estrategia dentro de la. RIED, 13(1), 191-208. doi:I.S.S.N.: 1138-2783

Franco, Y. (2017). Rol del Tutor en el Contexto del Aprendizaje Virtual. Revista Scientific, 2(6), 270-285. doi:doi.org/10.29394/scientific.issn.25422987.2017.2.6.14.270-285

Freire, P. (1996). Pedagogía de la autonomía. Buenos Aires: Grupo Editorial Siglo Veintinuo. Obtenido de ISBN: 978-987-629-039-5

Gairín, J., Feixas, M., Guillamón, C., \& Quinquer, D. (2004). La tutoría académica en el escenario europeo de la Educación Superior. Dialnet(49), 61-67. doi:ISSN: 0213-8646 
García, B., Ponce, S., García, M., Caso, J., Morales, C., Martínez, Y., . . . Islas, D. (2015). Las competencias del tutor universitario: una aproximación a su definición desde la perspectiva teórica y de la experiencia de sus actores. Scielo, 38(151), 140-122. Obtenido de http://www.scielo.org.mx/scielo.php?script=sci_arttext\&pid=S018526982016000100104

García-Valcárcel, A. (2008). La tutoría en la enseñanza universitaria y la contribución de las TIC para su. RELIEVE, 14(2), 1-14. Obtenido de http://www.uv.es/RELIEVE/v14n2/RELIEVEv14n2_3.htm

Garduño Vera, R. (2007). Caracterización del docente en la educación virtual: consideraciones para la Bibliotecología. Scielo, 21(43), 157-183. Obtenido de http://www.scielo.org.mx/scielo.php?script=sci_arttext\&pid=S0187358X2007000200007

Gómez-Collado, M. E. (2012). La percepción de los estudiantes sobre el Programa de Tutoría Académica. Convergencia, 19(58), 209-233. doi:versión On-line ISSN 2448-5799versión impresa ISSN 1405-1435

Gonzalo, N. (2020). La tutoría en las carreras de educación. Padres Y Maestros / Journal of Parents and Teachers(381), 54-60. doi:https://doi.org/10.14422/pym.i381.y2020.008

Hernández, J., Tobón, S., Gonzalez, L., \& Guzmán, C. (2015). Evaluación socioformativa y rendimiento académico en un programa de posgrado en línea. Paradigma, 36(1), 30-41. doi:http://ve.scielo.org/pdf/pdg/v36n1/art03.pdf

Lobato Fraile, C., \& Guerra Bilbao, N. (2016). La tutoría en la educación superior en Iberoamérica: Avances y desafíos. Redalyc, 379-398. doi:0211-819X

Maluenda, J., Moraga, F., \& Díaz, A. (2019). I rol del estudiante en elfenómeno delCompromiso Académico en Educación Superior. Rev. Estud. de Psic. y Cienc. Soc, 14(1), 81-94. doi:https://revistas.ucr.ac.cr/index.php/wimblu/article/view/35876/36850

Medina Alfonso, I. (2015). Las Funciones del Tutor de la Carrera de Pedagogía Psicología. Redalyc, 3(31), 32-44. Obtenido de https://www.redalyc.org/pdf/4780/478047207004.pdf

Mele, A. (2017). La tarea del tutor como acompañante del aprendizaje del alumno. En Reflexión Académica en Diseño y Comunicación NoXXXII (Vol. 32, págs. 190192). Buenos Aires. doi:ISSN: 1668-1673

Núñez Naranjo, A. F., Becerra García, E. B., \& Olalla Pardo, V. E. (2021). Autogestión del aprendizaje: Revisión de la literatura. Explorador Dlgital, 5(2), 6-22. doi:https://doi.org/10.33262/exploradordigital.v5i2.1649

Núñez-Naranjo, A. (2019). Deserción y estrategias de retención: un análisis desde la universidad particular. 593 Digital Publisher CEIT, 5((5-2)), 78-87. doi:https://doi.org/10.33386/593dp.2020.5-2.306

Núñez-Naranjo, A. (2020). Deserción y retención: retos en la educación superior. Revista Científica Retos de la Ciencia, 4(2), 15-23. Obtenido de https://retosdelacienciaec.com/Revistas/index.php/retos/article/view/320

Obaya, A., \& Vargas, Y. (2014). La tutoría en la educación superior. Educación Química, 25(4), 478-487. Obtenido de http://www.scielo.org.mx/scielo.php?script=sci_arttext\&pid=S0187893X2014000400012

Pagano, C. (2008). Los tutores en la educación a distancia. Un aporte Teórico. Revista de Universidad y Sociedad del Conocimiento, 1-11. Obtenido de 
https://rusc.uoc.edu/rusc/ca/index.php/rusc/article/download/v4n2pagano/304-1220-2-PB.pdf

Ponce Ruiz, D. V. (2021). Modelo de gestión de tutorías para la contribución de la formación profesional de los estudiantes de Derecho en la Universidad Regional Autónoma de Los Andes, Quevedo. REVISTA CONRADO, 17(S1), 251-257. doi:ISSN: 1990-8644

Quisel, E., \& Campana Concha, A. (2020). Tutoría docente y competencia académica en los estudiantes $X$ semestre, Escuela Profesional de Educación Física, Facultad de Educación - UNMSM 2018. Big Bang Faustiniano, 9(3), 28-32. doi:https://doi.org/10.51431/bbf.v9i3.639

Saiz, A. (2021). Una investigación sobre tutorías compartidas. REOP, 31(3), 41-58. doi:ISSN electrónico: 1989-7448

Salazar-Gómez, E., Tobón, S., \& Juárez-Hernández, L. (2018). Diseño y validación de una rúbrica de evaluación de las competencias digitales desde la socioformación. Revista de Investigación Apuntes Universitarios, 8(3), 24-42. doi:DOl:https://doi.org/10.17162/au.v8i3.329

Sánchez, J. (2015). El rol del tutor en la educación Superior a distancia. Virtualmente, 3(1), 22-34. Obtenido de https://journal.universidadean.edu.co/index.php/vir/article/view/1428

Silva Quiroz, J. (2010). El rol del tutor en los entornos virtuales de aprendizaje. Redalyc, 13-23. Obtenido de https://www.redalyc.org/pdf/1794/179420763002.pdf

Tapia, F., Lara, D., Aranda, A., \& Naranjo, A. (2018). Modelo Educativo. Quito: Universidad Tecnológica Indoamérica.

Tobón, S. (2014). Metodología de gestión curricular : una perspectiva socioformativa. Mexico: Trillas. doi:ISBN_978-607-17-1533-5

Universidad Tecnológica Indoamérica. (07 de 02 de 2018). Lex-UTI. Obtenido de Reglamento del sistema de evaluación estudiantil de grado y posgrado: https://www.uti.edu.ec/ utiweb/wpcontent/uploads/2018/04/Digitalizaci\%C3\%B3n-r\%C3\%A1pida-a-color-a-unarchivo-PDF_14-1.pdf 